\title{
EchoGéo
}

33 | 2015

Activisme, participation, contestation : la place des habitants dans les processus de patrimonialisation en périphéries urbaines

\section{Le quartier de l'Arsenal, un " village dans la ville » entre patrimonialisation et normalisation}

Quand la municipalité prend la main sur la mémoire ouvrière

\section{Diego Miralles Buil}

\section{(2) OpenEdition}

1 Journals

\section{Electronic version}

URL: https://journals.openedition.org/echogeo/14326

DOI: $10.4000 /$ echogeo. 14326

ISSN: 1963-1197

\section{Publisher}

Pôle de recherche pour l'organisation et la diffusion de l'information géographique (CNRS UMR 8586)

\section{Electronic reference}

Diego Miralles Buil, "Le quartier de l'Arsenal, un « village dans la ville » entre patrimonialisation et normalisation ", EchoGéo [Online], 33 | 2015, Online since 30 September 2015, connection on 07 December 2022. URL: http://journals.openedition.org/echogeo/14326 ; DOI: https://doi.org/10.4000/ echogeo.14326

This text was automatically generated on 11 August 2021.

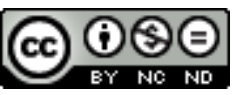

Creative Commons - Attribution-NonCommercial-NoDerivatives 4.0 International - CC BY-NC-ND 4.0 https://creativecommons.org/licenses/by-nc-nd/4.0/ 


\title{
Le quartier de l'Arsenal, un « village dans la ville » entre patrimonialisation et normalisation
}

Quand la municipalité prend la main sur la mémoire ouvrière

\author{
Diego Miralles Buil
}

\section{Introduction}

1 Cette contribution propose d'analyser le rôle que peuvent prendre les municipalités dans la dynamisation d'une "participation habitante» dans un contexte de patrimonialisation d'une cité ouvrière. Elle se basera sur le cas de la cité ouvrière de l'Arsenal, construite par l'État français en 1917. Celle-ci est située à cheval entre la ville moyenne de Roanne et la commune de Mably, toutes deux situées dans le département de la Loire, et possédant un fort passé industriel.

2 Les "cités ouvrières " correspondent à des ensembles de logements groupés ou pavillonnaires construits par une même entreprise dans le but d'y loger ses employés. La majorité de ces logements furent construits au début du XXe siècle, souvent dans une perspective hygiéniste et paternaliste (Duchêne, Langumier, Morel Journel, 2010, p. 10). Ces dernières décennies, en France, ces formes urbaines ont pour la plupart subi de grands bouleversements. En effet, la plupart des industries qui leur ont donné vie se sont retirées et le lien entre entreprise et logements disparait de plus en plus (Duchêne, 2010a). Se pose donc la question du devenir de ces cités ouvrières (Duchêne, Langumier, Morel Journel, 2010, p. 16).

3 Au sein de l'ouvrage collectif Cités ouvrières en devenir, Ethnographies d'anciennes enclaves industrielles qu'il a dirigé, François Duchêne propose de " remettre les habitants au premier plan " (Duchêne, Langumier, Morel Journel, 2010, p. 11). Dans un travail concernant la cité des Combes au Chambon-Feugerolles et à La Ricamarie, Christelle Morel Journel interroge les notions de normalisation et de valorisation de ces formes urbaines en ouvrant sur la question d'une possible construction patrimoniale (Morel Journel, 2010, 
p. 142). En effet, la plupart des cités ouvrières subissent actuellement des processus de normalisation (normalisation du bâti, homogénéisation sociale, etc.) tendant à effacer leur spécificité ouvrière, et très peu font l'objet de patrimonialisation. Selon Vincent Veschambre, le virage des années 1970 en matière de patrimonialisation avait pour but "d'intégrer le monument de classe dans le patrimoine de tous", et d'ajouter que "le monument incite d'abord à se souvenir des puissants» (Veschambre, 2002, p. 65). De plus, l'industrie du $\mathrm{XIX}^{\mathrm{e}}$ et $\mathrm{XX}^{\mathrm{e}}$ siècle a contribué à générer ou à entretenir des idéologies telles que le communisme et l'anarcho-syndicalisme. Ainsi, selon François Duchêne, du fait de la subversivité de cette industrie, les «classes dominantes" semblent vouloir banaliser ses traces, voire les effacer totalement (Duchêne, 2005, p. 518). Or, les ouvriers ou descendants d'ouvriers n'accèdent que rarement aux postes d'administration supérieure leur permettant d'avoir un poids dans les décisions en matière de patrimonialisation ${ }^{1}$. Ainsi, cette normalisation peut parfois mener à des conflits entre institutions et habitants. Ces derniers revendiquent parfois un maintien de la «mémoire collective» du quartier (Halbwachs, 1997) à travers la patrimonialisation de ce dernier. Mais les institutions s'emparent aussi parfois de «l'argument patrimonial» afin de valoriser un quartier urbain dans un but souvent caché de marketing territorial (Debos, 2012).

4 À travers le cas d'étude des cités ouvrières de l'Arsenal de Roanne, nous aborderons dans cet article la question de la valorisation ou de la normalisation des cités ouvrières et analyserons les forces en présence pouvant favoriser la patrimonialisation de ces entités urbaines. Par normalisation nous entendons leurs «remises aux normes, techniques et sociales" (Duchêne, Langumier, Morel Journel, 2010, p.13), c'est-à-dire leur homogénéisation spatiale, architecturale et sociale par rapport aux autres espaces urbains alentour. Autrement dit, ce concept pointe l'effacement de la spécificité de ces cités ouvrières, qui est due à leur statut d'espace habité par les travailleurs d'une même entreprise et géré par celle-ci. Inversement, le concept de valorisation de ces cités ouvrières met en lumière les processus tendant à rappeler, réhabiliter ou encore favoriser le maintien de l'héritage (social mais aussi architectural) de ces «isolats urbains " (Duchêne, 2005). Cette valorisation peut être symbolique (rappel de la mémoire ouvrière d'une cité lors d'expositions), architecturale, foncière, etc. (Veschambre, 2005). Ces concepts de valorisation et de normalisation peuvent également s'appréhender à travers les processus de territorialisation, déterritorialisation et reterritorialisation (Zanetti, 2012, p. 24). La territorialisation - vue comme " processus de transformation de l'espace en territoire" - induit "une approche dynamique de l'appropriation de l'espace disponible pour la réalisation intentionnelle d'un objectif, celui de la structuration d'un territoire» (Zanetti, 2012, p. 24). Ainsi, le territoire est vu comme "un processus en perpétuelle évolution" (Zanetti, 2012, p. 24). De ce fait, les acteurs agissant dans la construction des territoires engendreraient des processus de territorialisation/ déterritorialisation/reterritorialisation. La déterritorialisation, loin d'être vue comme la disparition du territoire, témoigne d'un réagencement territorial, d'une "ré-énonciation d'un mode de territorialisation d'une instance ou d'un système d'action donnés" (Vanier, 2006). De la même manière, la reterritorialisation ne signifie pas un retour vers un territoire passé mais bien "une transformation des anciennes configurations territoriales" (Zanetti, 2012, p. 27). Ainsi, le cycle de territorialisation/déterritorialisation/ reterritorialisation témoigne d'une recomposition territoriale sur la longue durée en fonction de l'intentionnalité des acteurs en présence. Ce cycle peut permettre d'éclairer les processus de valorisation ou de normalisation des cités ouvrières. En effet, la 
normalisation d'une cité ouvrière peut être vue comme une déterritorialisation dans le sens où elle va "gommer " la spécificité ouvrière du lieu (Duchêne, Langumier, Morel Journel, 2013, p. 37). Au vu de la situation géographique très spécifique des cités ouvrières de l'Arsenal de Roanne - à cheval entre les communes de Mably et de Roanne - nous nous interrogerons tout particulièrement sur le rôle des municipalités au sein de ces processus ainsi que sur la place des habitants dans la valorisation de la mémoire ouvrière de cet espace.

Pour mener à bien cette étude, nous nous appuierons sur un travail de terrain réalisé en octobre 2013, basé sur une observation et sur la mise en place d'une vingtaine d'entretiens d'acteurs. Ces entretiens furent notamment effectués auprès des habitants des cités ouvrières de l'Arsenal (anciens et nouveaux venus), des membres des municipalités de Roanne et de Mably, ainsi que des membres d'associations implantées dans les cités de l'Arsenal'2 .

6 Nous commencerons par présenter l'histoire de la construction de l'Arsenal, menant à la territorialisation de ce que nous appellerons «le territoire de l'Arsenal $»^{3}$. Nous aborderons ensuite le processus de déterritorialisation de cet ensemble urbain dû au désengagement de l'État et à l'ouverture du quartier au marché privé de l'immobilier. Ce désengagement explique en partie la forte division socio-spatiale du quartier d'aujourd'hui. Enfin, nous aborderons le mécanisme de normalisation différenciée qui a lieu au sein de ce quartier, ainsi que les forces sociales et politiques qui tentent d'infléchir ou au contraire d'accélérer cette normalisation.

\section{Roanne, Mably et l'Arsenal : de la territorialisation à la déterritorialisation d'une cité ouvrière}

7 Roanne est une ville moyenne de 36147 habitants (en 2011) dont l'industrie s'est tout particulièrement développée durant le XIX ${ }^{\mathrm{e}}$ siècle autour de l'industrie textile (Houssel, 1979). La commune a également vu son industrie prendre un nouveau tournant en 1916 avec l'arrivée de l'Arsenal de Roanne, un atelier de construction militaire. La ville de Mably quant à elle - jouxtant celle de Roanne (illustration 1) - compte 7603 habitants en 2011. Son histoire industrielle est fortement liée à celle de Roanne mais son organisation spatiale est assez atypique car elle comporte quatre quartiers principaux non contigus (illustration 2). Tout comme sa voisine roannaise, Mably a connu une période de forte industrialisation, notamment par le biais de l'industrie textile et de l'Arsenal. 
Illustration 1 - Roanne et Mably dans le département de la Loire

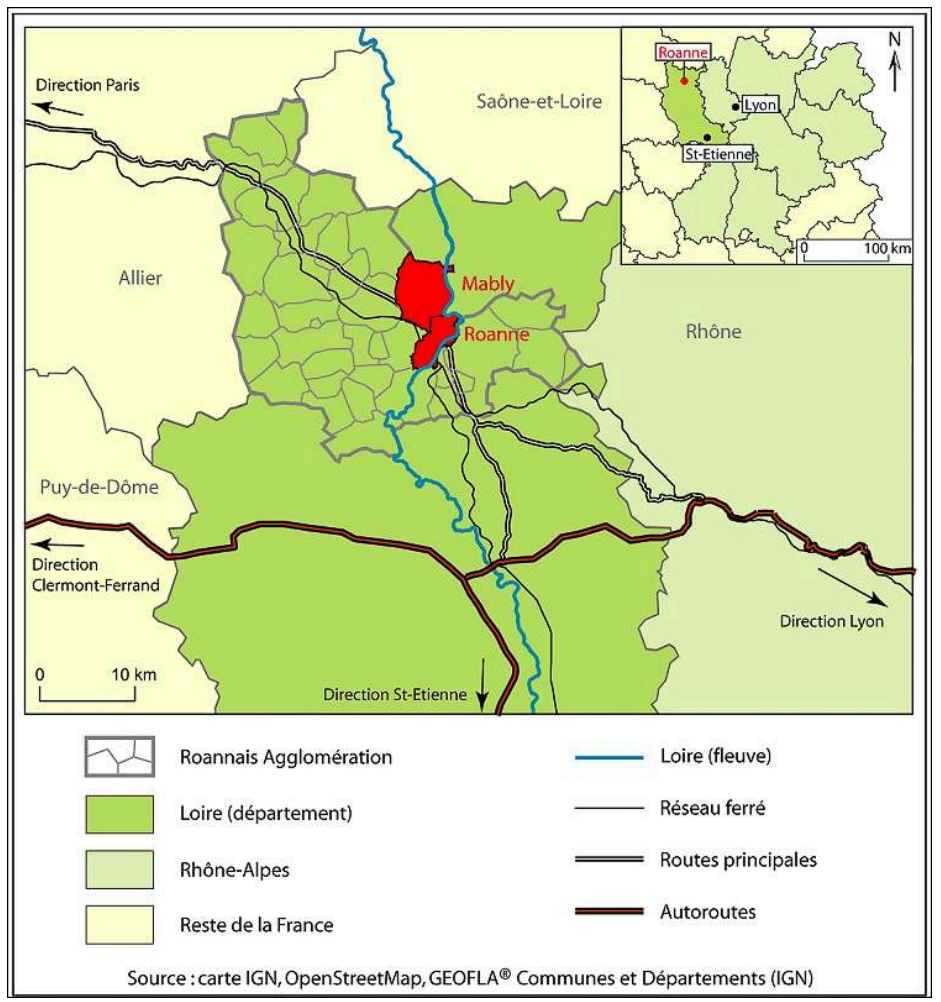

Auteur : D. Miralles Buil, 2014.

Illustration 2 - Les quatre grands quartiers de Mably : le Bourg, les Tuileries, les Sables et l'Arsenal

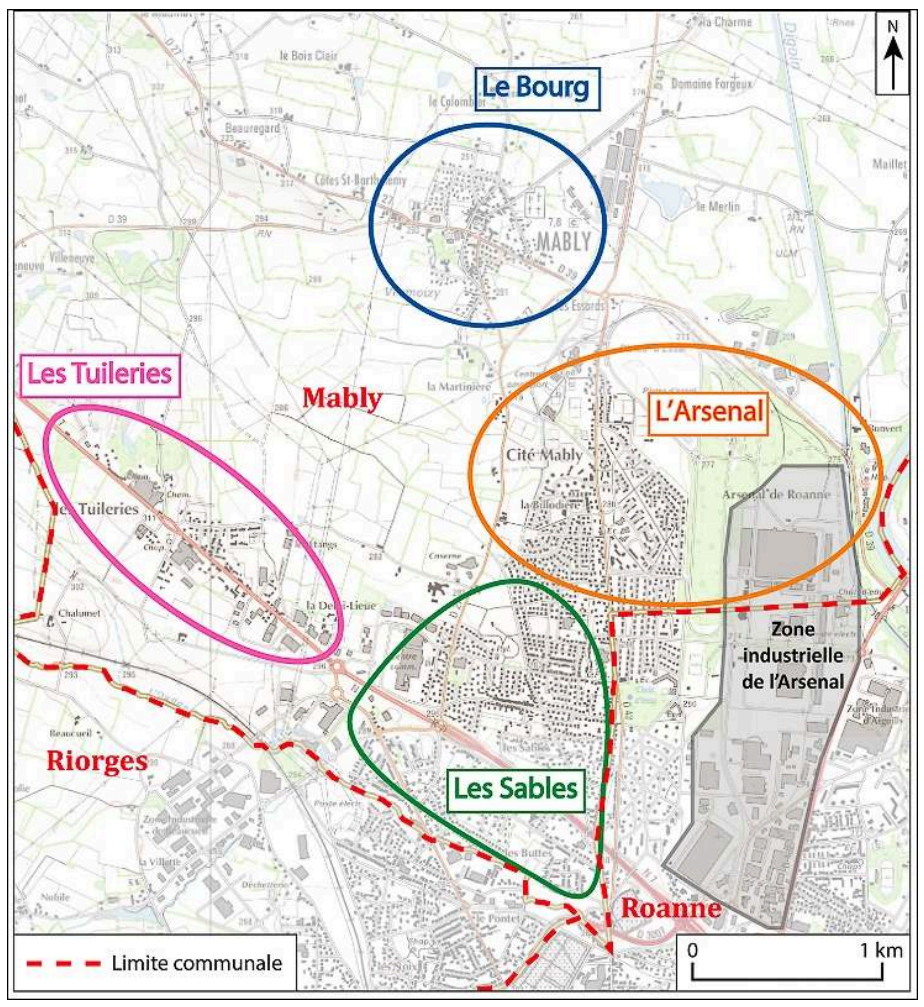

Source : Carte IGN, http://www.ville-mably.fr/presentation.html, consulté le 10 avril 2014 ; auteur : D. Miralles Buil, 2014 


\section{L'Arsenal de Roanne : des obus aux tracteurs}

8 L'Arsenal de Roanne fut construit par l'État français durant la Première Guerre mondiale. Aujourd'hui encore, certains de ses bâtiments sont toujours présents et témoignent de l'ancienne activité industrielle du territoire roannais.

9 En effet, l'Arsenal a été un véritable vecteur de développement des deux communes. Dès sa construction en 1916, il se concentre sur la production d'obus et de véhicules de guerre. Après la seconde guerre mondiale, il se tourne vers la production de tracteurs à usage agricole, sans pour autant arrêter les productions militaires (Barras, 1998). S'ensuit alors une baisse de son activité industrielle (dès 1980, les plans sociaux se multiplient) qui s'inscrit dans un contexte national plus large de désindustrialisation. Celle-ci a engendré de nombreuses mutations socio-spatiales sur les deux communes et particulièrement dans les anciennes zones industrielles comme le quartier de l'Arsenal.

\section{Une cité ouvrière spatialement divisée : du paternalisme au désengagement de l'Arsenal}

La spécificité de la cité ouvrière de l'Arsenal provient du fait qu'elle fut entièrement financée et construite par l'État français et non par une entreprise privée comme l'ont été la plupart des cités ouvrières à la même époque (Duchêne, Langumier, Morel Journel, 2010, p. 10). Pourtant, même si elle fut entièrement construite par le même acteur, elle n'en est pas pour autant spatialement et socialement homogène. En effet, du fait de la planification des travaux par l'état et de la topographie du terrain, cette cité fut divisée en trois ensembles bien distincts (illustration 3) qui ont joué un rôle dans les représentations du territoire par les habitants des cités. 
Illustration 3 - Les cités de l'Arsenal aujourd'hui

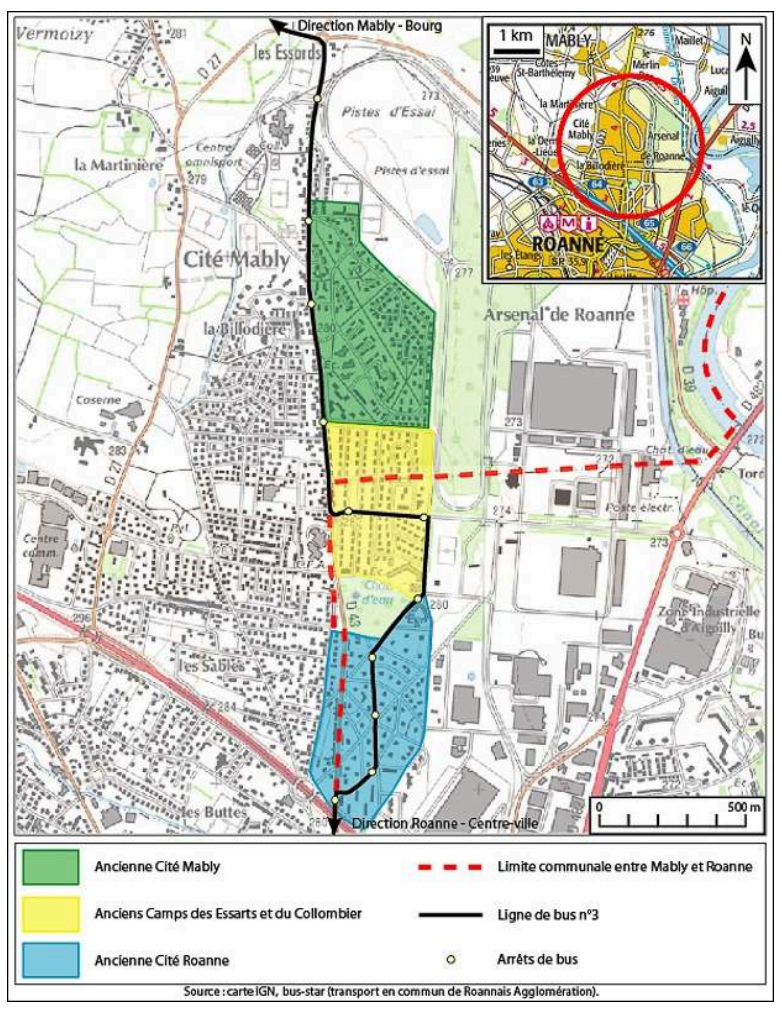

Auteur : D. Miralles Buil, 2014

\section{Une cité ouvrière financée par l'État français}

11 La construction de la cité ouvrière de l'Arsenal fut réalisée en deux temps. Il en découla donc une forte division socio-spatiale. En effet, en 1916, pour construire l'atelier de construction de l'Arsenal et sa cité ouvrière, l'État français réquisitionna de nombreuses fermes dont les terrains devinrent la propriété de l'État (Rocher, Trévarin, 2011, p. 78). Dès le projet de départ, l'État (à travers l'Arsenal, véritable entreprise étatique) prit en charge l'alimentation en eau, la création d'un tramway, d'un réseau d'égout, etc. Dans la perspective paternaliste et hygiéniste de l'époque, l'Arsenal planifia deux cités ouvrières différentes, la «Cité Roanne» et la «Cité Mably».

Conçues à deux époques différentes, les deux cités présentent une architecture contrastée de part et d'autre de la limite communale entre Roanne et Mably. En effet, la première cité ouvrière, celle de Roanne (construite fin 1917), fut d'abord basée sur le modèle de la "cité-jardin» (illustration 4) mais sa construction fut beaucoup plus longue que prévu. Aussi, pour la Cité Mably (construite en juin 1918), le modèle de logements choisi fut beaucoup plus simple comme en témoigne l'illustration 5 (Barras, 1998). De ce fait, dès leur construction, la Cité Roanne et la Cité Mably s'opposaient totalement sur le plan architectural, la simplicité des bâtiments de la Cité Mably tranchant fortement avec les majestueuses « villas » avec jardin de la Cité Roanne*.

À la diversité architecturale des deux grandes cités ouvrières de l'Arsenal s'ajouta une division socio-spatiale soulignant davantage le clivage entre les deux entités. Du point de vue spatial tout d'abord, la limite communément admise dans les représentations des acteurs de l'époque qui séparait les deux entités était matérialisée par «le petit 
bois ", un espace fortement arboré séparant la Cité Roanne au sud, de la Cité Mably au nord (voir le plan de l'exposition de la médiathèque de Mably - en note ci-dessus - ou encore l'illustration 3 ci-dessus). Dans ces représentations, aucune importance n'était donnée à la limite administrative des communes de Roanne et de Mably qui passait alors au beau milieu des camps des Essarts (voir l'illustration 3) et n'avait pas été prise en compte par l'État comme axe structurant de l'organisation spatiale des cités. Néanmoins, bien que non perçue par les acteurs de l'époque, cette limite administrative joua un rôle non négligeable dans le clivage social entre les deux cités ouvrières. En effet, durant les années de guerre, les ingénieurs militaires devaient respecter le cantonnement et n'avaient donc pas le droit d'habiter sur la commune de Mably. Ils ne pouvaient habiter qu'à Roanne. Ainsi, on retrouve chez beaucoup d'habitants de la Cité Mably un sentiment tendant à catégoriser la Cité Roanne comme étant «la cité des supérieurs ", autrement dit la cité des ingénieurs. Pourtant, la Cité Roanne n'abritait pas que des ingénieurs : certaines villas étaient également habitées par des techniciens ainsi que (plus rarement) par des ouvriers. Quoi qu'il en soit, cette limite administrative ajoutée à l'obligation pour les ingénieurs militaires de loger sur la commune de Roanne amplifia le clivage spatial déjà présent entre les deux cités en lui additionnant un clivage social. Effectivement, les habitants de la Cité Mably expriment toujours aujourd'hui un sentiment "de classe » tendant à affirmer que «les vrais ouvriers " habitaient la Cité Mably et que les « patrons » habitaient la Cité Roanne. Ce sentiment collectif est également partagé par les habitants de la Cité Roanne.

Illustration 4 - Une « cité-jardin » type de la Cité Roanne, construite en 1917

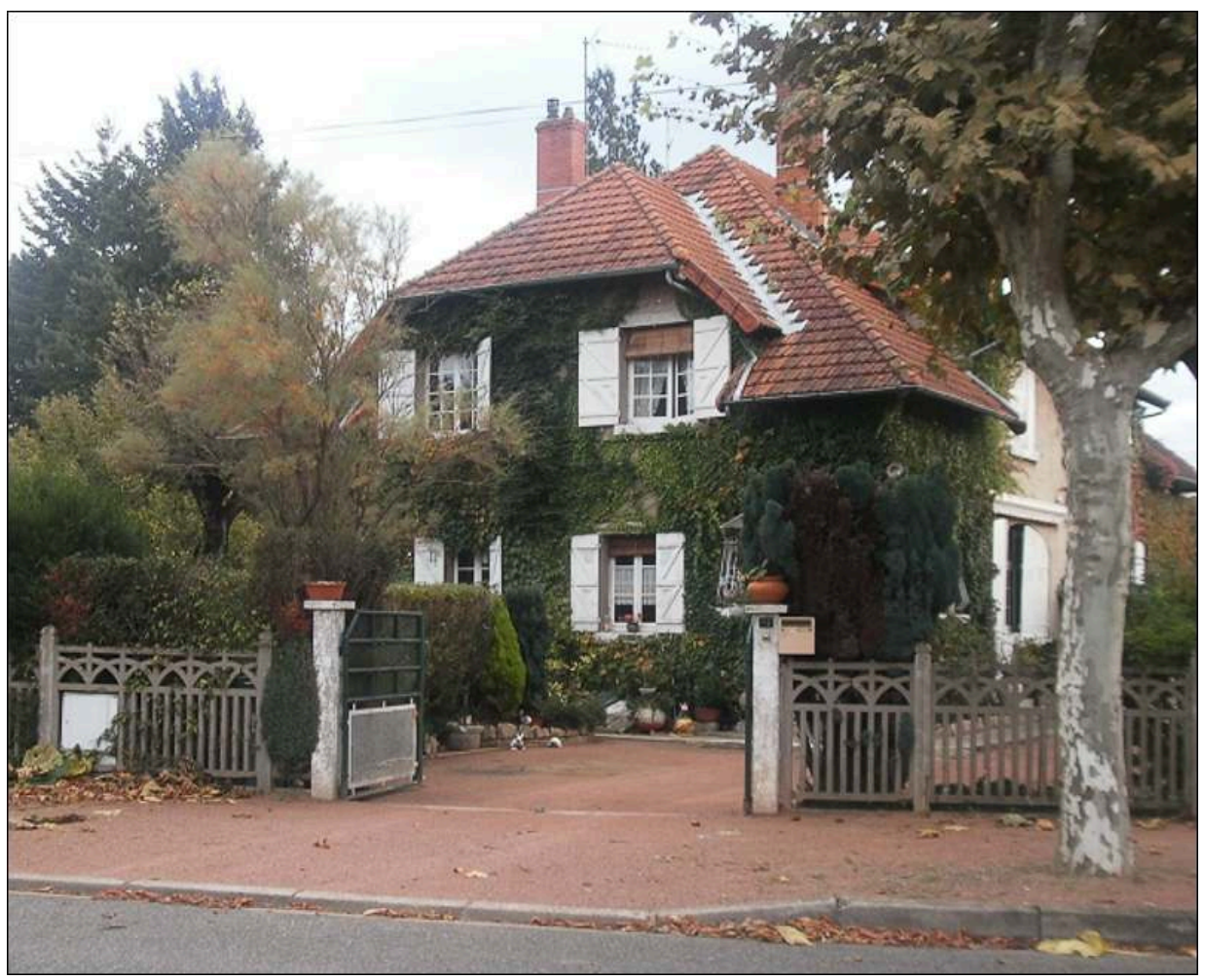

Auteur : D. Miralles Buil, octobre 2013. 
Illustration 5 - Les logements d'origine de la Cité Mably, construits en 1918

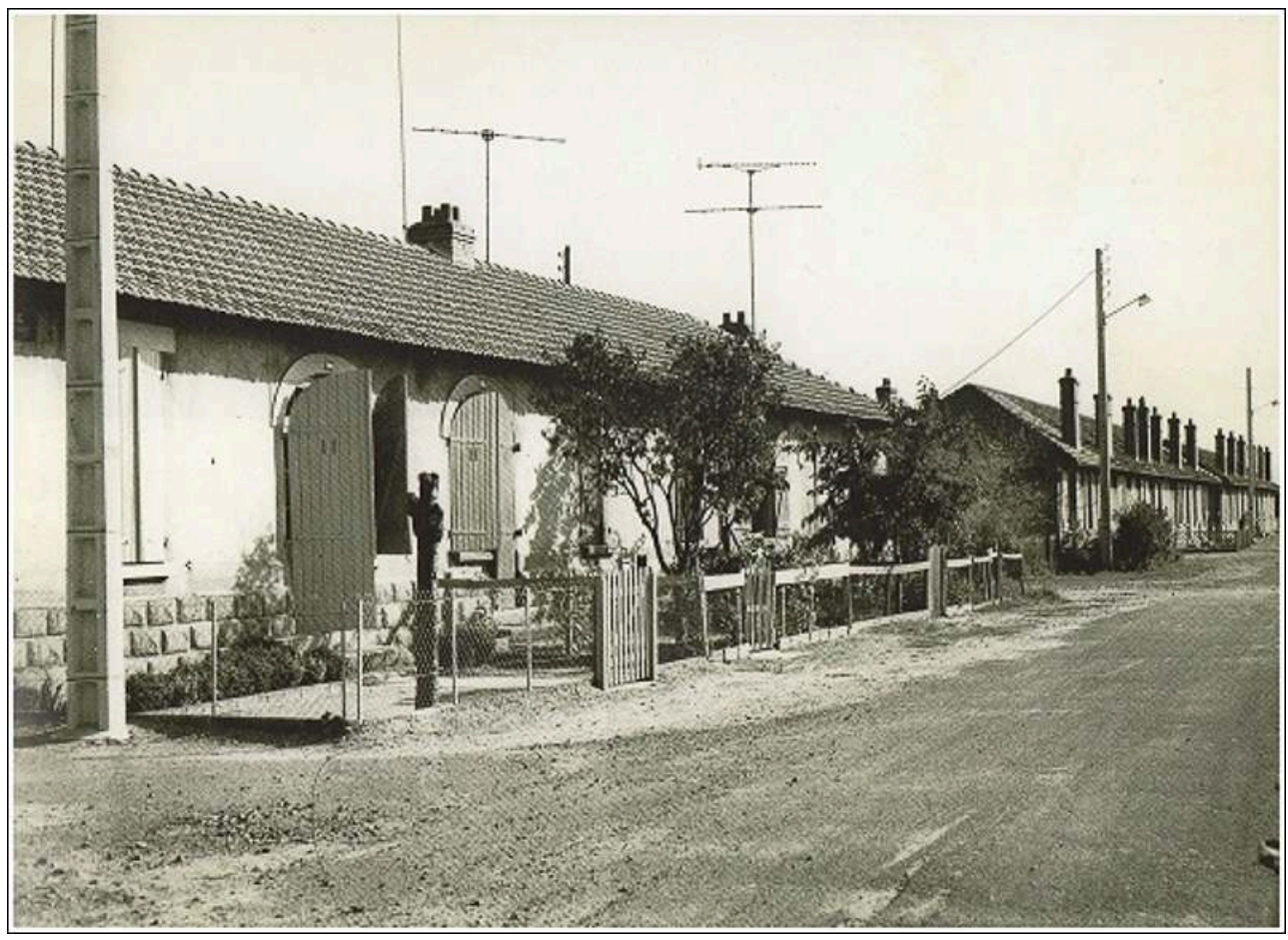

Source : Rocher, Trévarin, 2011, Mably, terre d'accueil, d'art et de solidarité. Roanne, Thoba's éditions, p. 91.

Du public au privé : le désengagement de l'Arsenal et la déterritorialisation des cités ouvrières

Malgré leur hétérogénéité architecturale et sociale, les cités ouvrières s'unifièrent autour du fait que l'Arsenal en était le gestionnaire et le lieu de travail de tous les habitants, le tout formant ce que l'on peut appeler le «territoire de l'Arsenal» (Zanetti, 2012, p. 15). Mais le désengagement de l'Arsenal (et donc de l'État) dès le début des années 1960 signa la fin de ce "territoire de l'Arsenal » et laissa les deux cités ouvrières à la charge de leurs municipalités respectives pour leur gestion et soumises aux règles du marché de l'immobilier.

Ce désengagement se réalisa en deux temps, témoignant des deux normalisations successives des cités ouvrières de l'Arsenal et mena à la déstabilisation du tissu urbain de ces cités. Premièrement, l'énorme besoin en logements qui se faisait ressentir au début des années 1960 poussa l'Arsenal à démolir les camps des Essarts et du Colombier afin de construire des logements ouvriers. C'est ainsi que dès 1962 fut construit un premier lotissement associatif basé sur le principe de l'accession à la propriété (Rocher, Trévarin, 2011, p. 159). Deux autres associations de constructeurs ont suivi, dont les logements furent construits au début des années 1970. L'illustration 6 présente ces logements tels qu'ils sont aujourd'hui. Cette vente aux travailleurs des terrains anciennement propriété de l'Arsenal - d'où un passage de statut de terrain public à celui de terrain privé - témoigne de la première normalisation du quartier de l'Arsenal ${ }^{5}$ (Duchêne, 2010b, p. 160). 
Illustration 6 - Un lotissement associatif construit à la place du camp du Colombier au début des années 70

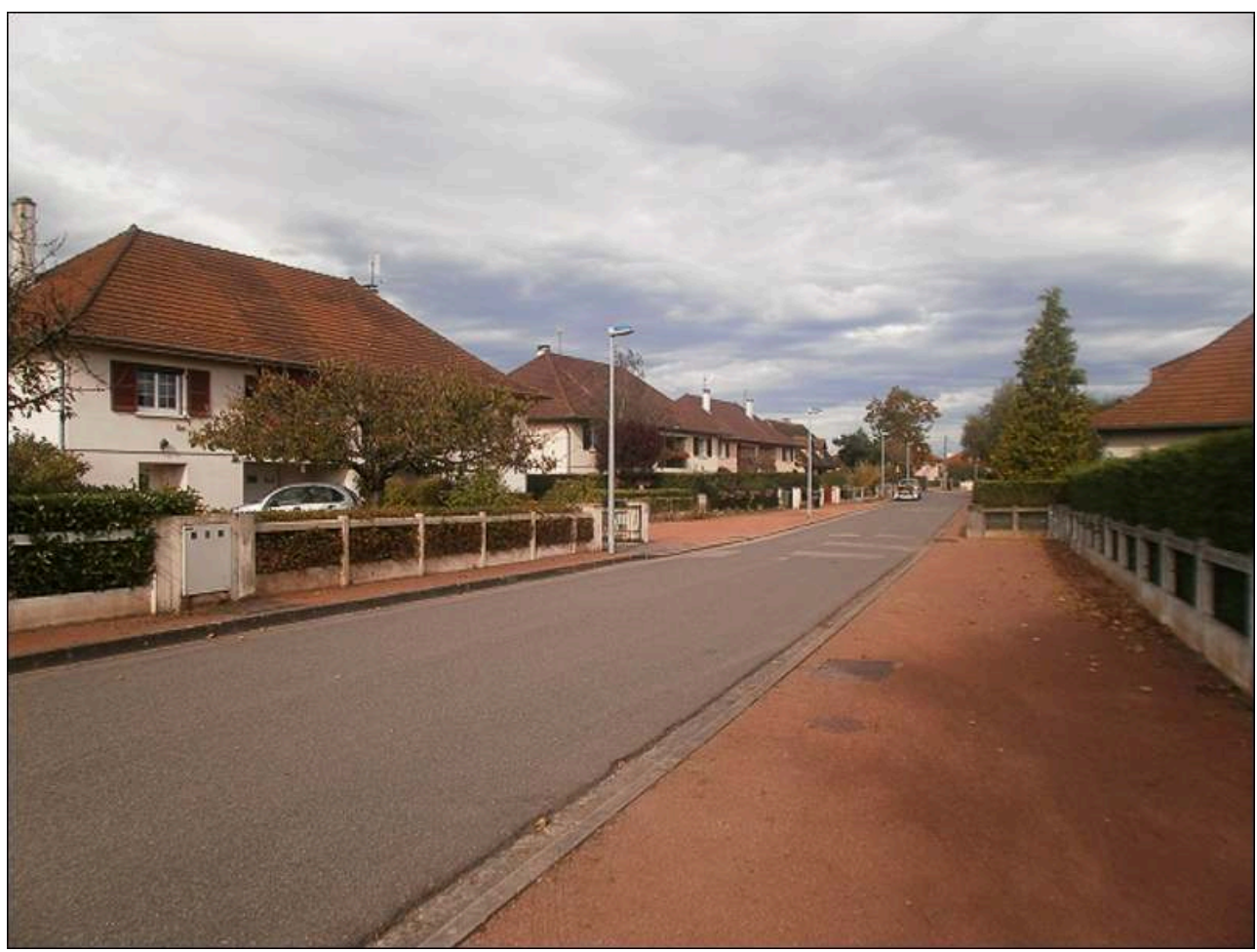

Auteur : D. Miralles Buil, octobre 2013.

La seconde normalisation des cités ouvrières de l'Arsenal se déroula au début des années 1970. La mauvaise qualité des bâtiments de la Cité Mably engendra rapidement leur détérioration, ce qui poussa l'Arsenal à les démolir entre 1972 et 1973 (Rocher, Trévarin, 2011, p. 161-162). Ce dernier ne souhaitait pas assurer la reconstruction de nouveaux logements sur le site et confia donc la construction de HLM à l'Office Public d'Aménagement et de Construction (OPAC) de Roanne ${ }^{6}$. De plus, la réglementation des Domaines fut modifiée et il ne fut plus possible de construire en tant qu'association. Ainsi, sur le site de l'ancienne Cité Mably (aux côtés des HLM), des lotissements furent construits et gérés par des sociétés immobilières (comme le Foyer Roannais par exemple). Les villas de la Cité Roanne quant à elles, de meilleure qualité, ne furent pas démolis, mais l'Arsenal les vendit en accession à la propriété à leurs occupants (Le Pays Roannais, 29/08/13). Ainsi, ce désengagement de l'Arsenal et cette entrée des logements sur le marché de l'immobilier signent la deuxième normalisation du quartier. On peut également y voir les prémisses d'un phénomène de déterritorialisation du «territoire de l'Arsenal ». Cela se caractérise ici par une rupture entre le territoire en question et l'organisation socio-spatiale et politique qui avait contribué à son élévation au rang de «territoire ».

Ainsi, la fracture - architecturale, historique et sociale - entre les deux cités ouvrières s'est encore fortement accentuée avec le désengagement de l'Arsenal et la rénovation des logements de la Cité Mably entreprise par des sociétés immobilières privées. Pour le cas de Mably, on peut donc déjà se questionner quant à la possible patrimonialisation d'une cité ouvrière dépourvue de ses logements ouvriers d'origine. 


\section{La « question patrimoniale » des cités de l'Arsenal : une gestion différenciée}

18 Avec la diminution de l'activité industrielle de l'Arsenal, plusieurs plans sociaux se succédèrent dès 1985 , entraînant le départ de nombreux ouvriers habitant le quartier. Cela affecta fortement la "mémoire collective» de ce dernier (Halbwachs, 1997). Aujourd'hui, les seuls garants de cette mémoire ouvrière restent les « anciens » ayant connu « le temps de l'Arsenal ». La plupart d'entre eux étant âgés, la mémoire collective du quartier risque donc de disparaitre avec eux. De plus, la perte du lien entre les habitants et l'usine d'armement pousse également à la normalisation du quartier. Pourtant, l'action de la municipalité peut jouer un rôle de catalyseur dans le maintien de cette mémoire collective.

\section{Le rôle des municipalités dans les processus de normalisation et de valorisation des cités ouvrières de l'Arsenal}

Le fort clivage entre les deux cités ouvrières de l'Arsenal s'est encore davantage amplifié avec le désengagement de l'Arsenal et la perte de la mémoire collective du quartier. Ainsi, la récupération de la gestion des cités par leurs municipalités respectives mena à une valorisation différenciée de ces entités urbaines. Ces municipalités disposent en effet de moyens à mettre au service de la mémoire collective que les habitants n'ont pas. Elles peuvent notamment accéder à des ressources leur permettant d'impulser ou de favoriser le développement d'une "volonté patrimoniale » chez les habitants.

La Cité Mably, du paternalisme social de l'Arsenal à la tutelle de la municipalité de Mably

La municipalité de Mably met actuellement en place une valorisation de la mémoire ouvrière de la Cité Mably. Pour cela, elle axe son action sur deux points : l'information et l'aménagement.

21 Premièrement, bien qu'à Mably les cités ouvrières d'origine aient presque toutes été démolies, la municipalité a réalisé un grand travail d'information destiné aux habitants de la commune. Le maire de Mably, très soucieux de la mémoire collective de sa commune, tente d'en valoriser le passé ouvrier en mobilisant plusieurs "outils patrimoniaux» et met en place plusieurs partenariats avec des institutions patrimoniales. Il s'agit par exemple d'expositions à la médiathèque municipale ${ }^{7}$, de la valorisation de documents historiques en partenariat avec le musée de Roanne ou encore de la publication d'un livre sur l'Histoire de Mably financé par la municipalité. Ce livre, intitulé Mably terre d'accueil, d'art et de solidarité, publié en 2011 et comportant de nombreuses pages traitant des cités ouvrières de l'Arsenal, a été distribué dans plusieurs bibliothèques françaises ${ }^{8}$. La municipalité de Mably encourage également l'élaboration d'un second ouvrage du même type exclusivement axé sur les cités ouvrières de l'Arsenal, ouvrage qu'elle se propose de soutenir financièrement. Cette activité de valorisation historique de l'équipe municipale témoigne bien de la volonté de cette dernière de maintenir active cette mémoire ouvrière, et cela même si les logements ouvriers de la Cité Mably n'existent plus. 
Deuxièmement, la mairie de Mably tente de préserver l'ancienne organisation sociale du quartier à travers son aménagement. Cette démarche s'inscrit dans le prolongement des décisions prises en 1976 par la municipalité de Mably. En effet, dès la reconstruction des logements sur le site de la Cité Mably en 1976-1977, la municipalité (PCF) de l'époque décida de conserver l'ancienne trame viaire de la Cité Mably telle qu'elle était à l'origine et de la réserver à un usage piéton et cycliste. À travers la préservation de cet axe structurant de la Cité Mably, l'objectif de la municipalité était alors de conserver une organisation sociale particulière et de rappeler l'origine ouvrière du quartier. Cet espace est aujourd'hui encore utilisé par les habitants comme un véritable jardin public, comme en témoignent les illustrations 7 et 8. Depuis, les municipalités successives de Mably ont pérennisé cette idée en érigeant ce "parc urbain " au rang de véritable espace public et de mémoire (illustration $9^{9}$ ). De plus, l'actuelle municipalité de Mably (PS) a décidé de réhabiliter les deux seuls bâtiments d'origine de la Cité-Mably n'ayant pas été démolis en 1972-1973. Ils abritent aujourd'hui le pôle Enfance-Famille de la commune (illustration 10). La réhabilitation de ces deux bâtiments - "pour la mémoire des générations futures » d'après le maire de Mably est un signal fort de la municipalité. Il s'agit donc ici, à travers l'aménagement, de protéger un type d'organisation sociale et paysagère datant de l'époque des cités de l'Arsenal, notamment afin de rappeler l'origine ouvrière du quartier et de la municipalité de Mably (PCF puis PS). Nous sommes donc bien, dans les faits, en présence d'une patrimonialisation du paysage plaçant l'espace de la Cité Mably « hors de l'emprise du temps » (Coanus, 1995).

Illustration 7 - Les rues piétonnes de la Cité Mably aménagées par la mairie de Mably

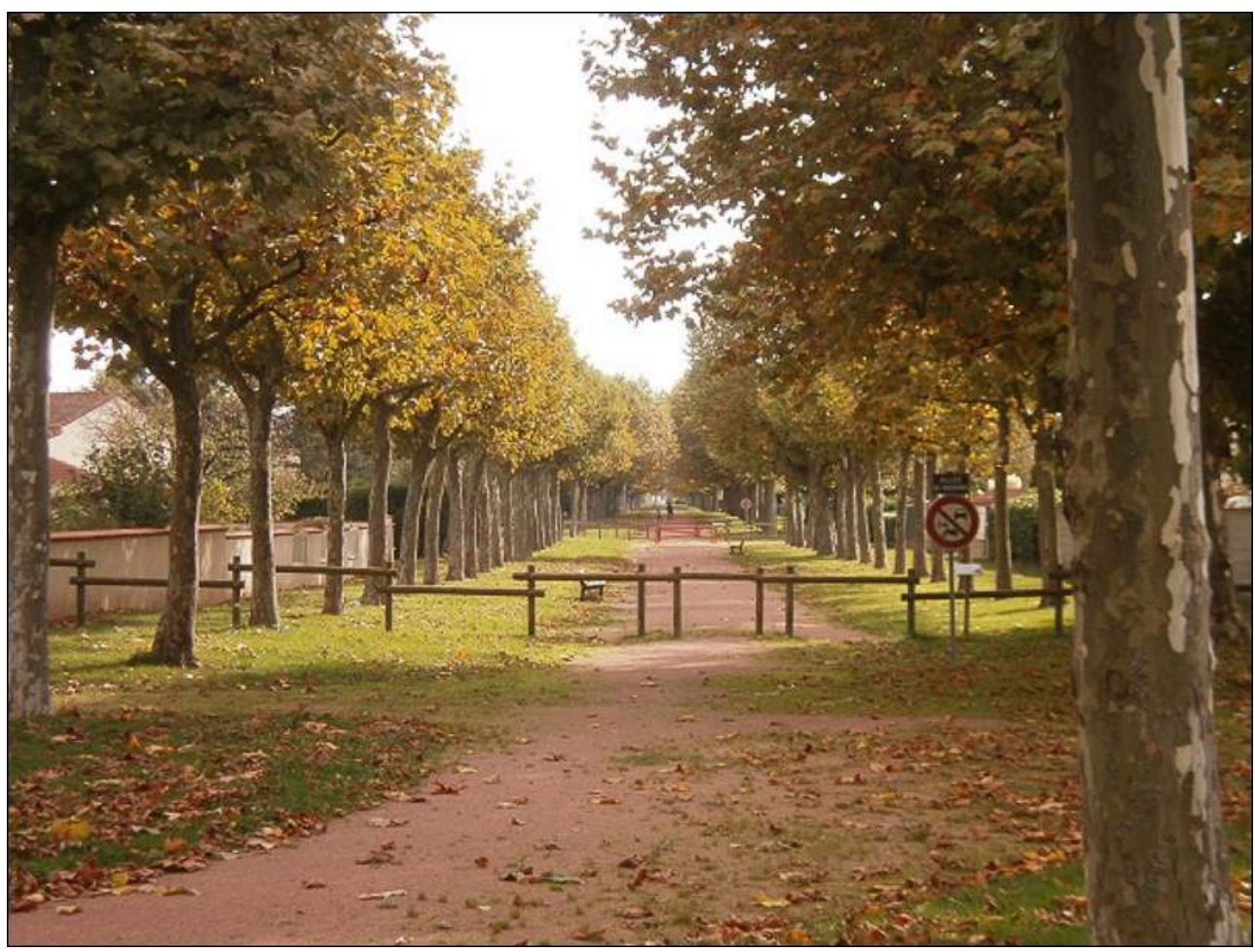

Auteur : D. Miralles Buil, octobre 2013. 
Illustration 8 - L'Allée de Soissons de la Cité Mably aménagée en véritable jardin public

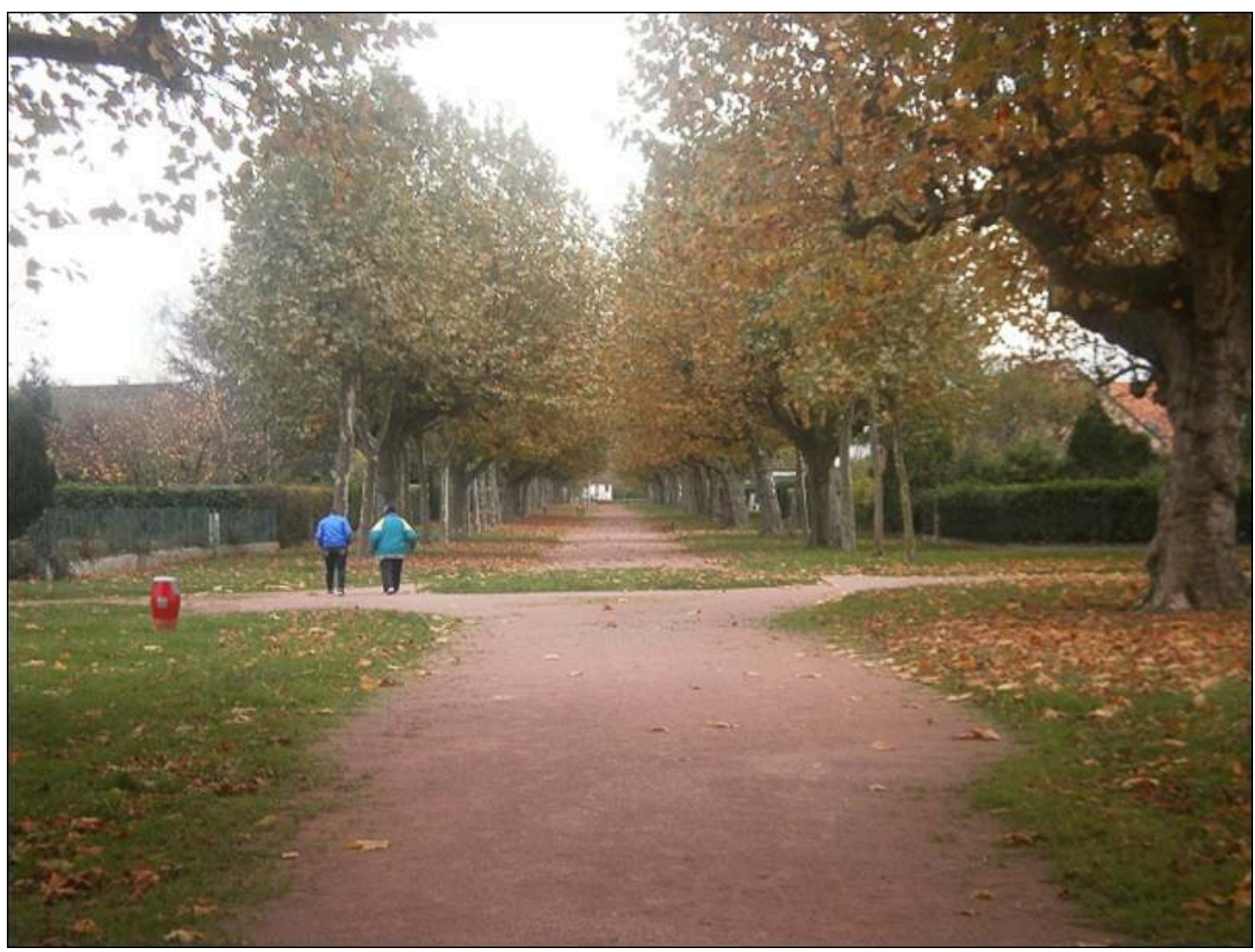

Auteur : D. Miralles Buil, octobre 2013.

Illustration 9- La trame viaire de la Cité Mably d'aujourd'hui sur photographies aériennes de 1947

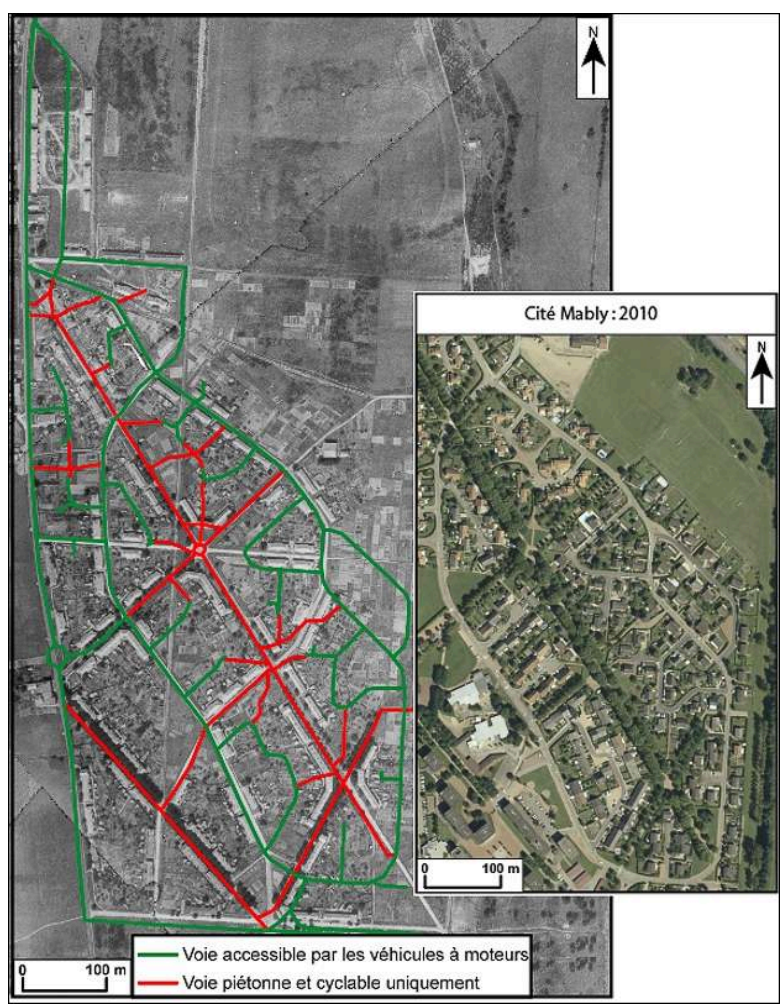

Auteur : D. Miralles Buil, octobre 2013. 
Illustration 10 - Les deux derniers bâtiments de la Cité Mably d'origine ayant été réhabilités, Rue de Guise à Mably

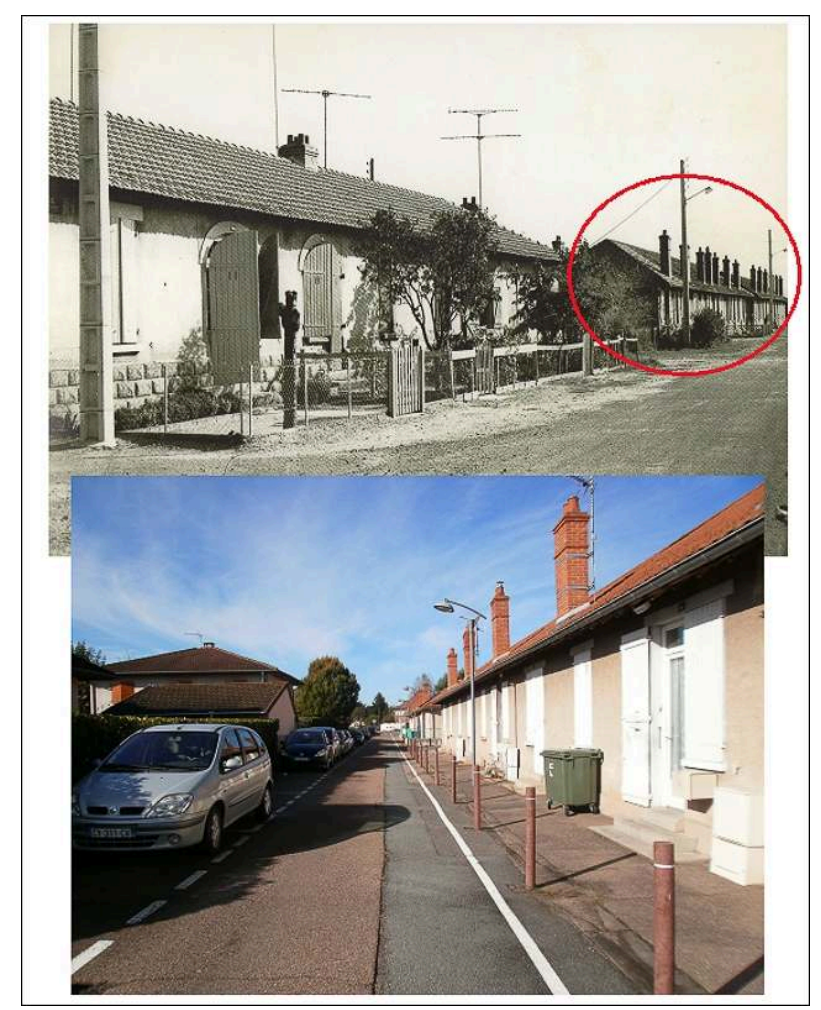

Source : Rocher, Trévarin, 2011, p. 91 ; auteur : D. Miralles Buil, octobre 2013.

Par conséquent, cette politique volontariste menée par la municipalité nous amène à penser que cette dernière tente d'alimenter un processus de reterritorialisation de la Cité Mably. Il est également possible de voir dans la politique de valorisation du quartier menée par la municipalité le prolongement ou le maintien d'une forme de paternalisme, rôle qu'endossait l'Arsenal avant son désengagement. À la manière d'un "conservateur de musée », le maire de Mably et son équipe tentent de préserver l'objetpatrimonial que serait la Cité Mably d'antan (Coanus, 1995). Mais la municipalité n'en est pas pour autant "propriétaire» (Coanus, 1995). Selon la mairie de Mably, le propriétaire - le sujet collectif de cet objet-patrimoine - resterait les habitants actuels de la Cité Mably. Mais qu'en est-il de ces actuels habitants ? Sont-ils réceptifs à cette valorisation de la mémoire ouvrière de leur quartier? Et tout d'abord, qui sont les habitants de l'actuelle Cité Mably?

Les habitants de l'actuelle Cité Mably ne sont pas tous des «anciens du temps de l'Arsenal ». Nombreux sont les nouveaux habitants vivant sur le site de l'ancienne Cité Mably qui n'ont jamais réellement eu conscience de l'histoire ouvrière de ce quartier. En effet, dès les années 1980 , le départ pour cause de licenciement de nombreux ouvriers ayant vécu et travaillé à l'Arsenal engendra un déclin de la mémoire ouvrière du quartier. De plus, l'année 1977correspond à l'arrivée des équipes municipales de gauche sur l'agglomération roannaise (Jean Auroux (PS) à Roanne, Bernard Jayol (PS) à Riorges et Paul Desroches (PCF) à Mably). Dès leur élection, ces maires mirent en place une politique volontariste de développement urbain. Celle-ci a fortement brassé les populations du territoire, impactant ainsi la mémoire collective du quartier. En effet, le «foyer ouvrier de l'Arsenal », toujours présent en 1977 malgré le désengagement de 
l'Arsenal, a été "éclaté» par cette politique qui a ainsi séparé les habitants en permettant à d'autres de s'installer dans le quartier. De plus, les politiques urbaines plus récentes favorisant la mixité sociale ont également contribué au mélange de la population et ainsi à l'affaiblissement de la mémoire collective des habitants du quartier en diminuant le nombre d'anciens habitants. Selon l'actuel président de l'Amicale Laïque de l'Arsenal, bien que la mairie de Mably tente de rapprocher les différentes générations, les jeunes ne sont pas intéressés par le maintien de la mémoire ouvrière du quartier. Les seuls garants de cette mémoire ouvrière restent donc les " anciens », mais ceux-ci ne sont aujourd'hui plus majoritaires dans la Cité Mably et ne semblent pas réellement concernés par le maintien de cette mémoire. "J'aime ce quartier, mais on ne pourra pas rester comme ça longtemps. Le monde change, maintenant on peut se déplacer à Roanne facilement, et c'est bien " affirme une retraitée de l'actuelle Cité Mably y habitant depuis plus de trente ans. Son époux ajoute : «Je pense qu'avec le temps la mémoire ouvrière du quartier va entièrement s'effacer. Quand nous serons partis, malgré tous les livres d'Histoire et les expositions que la mairie mettra en place, cela ne sera que de l'histoire ancienne... C'est dur à admettre pour nous parce qu'il s'agit de notre histoire. Mais ce n'est pas forcément un mal... il faut laisser la place aux jeunes comme on dit !... ».

\section{La Cité Roanne, une cité ouvrière en voie de normalisation}

La Cité Roanne (la cité ouvrière de l'Arsenal côté Roanne) quant à elle, n'a pas été entièrement démolie. Il reste encore la plupart des anciennes villas en état d'origine comme en témoigne l'illustration 11 ci-dessous. Certaines de ces villas sont aujourd'hui séparées en deux parties (voire en quatre) dans lesquelles vivent autant de ménages et fonctionnent alors sous un modèle de copropriété. Pour préserver cette architecture, on peut donc supposer que la municipalité de Roanne a été amenée à les valoriser d'une manière ou d'une autre. Mais en fait il n'en est rien. 


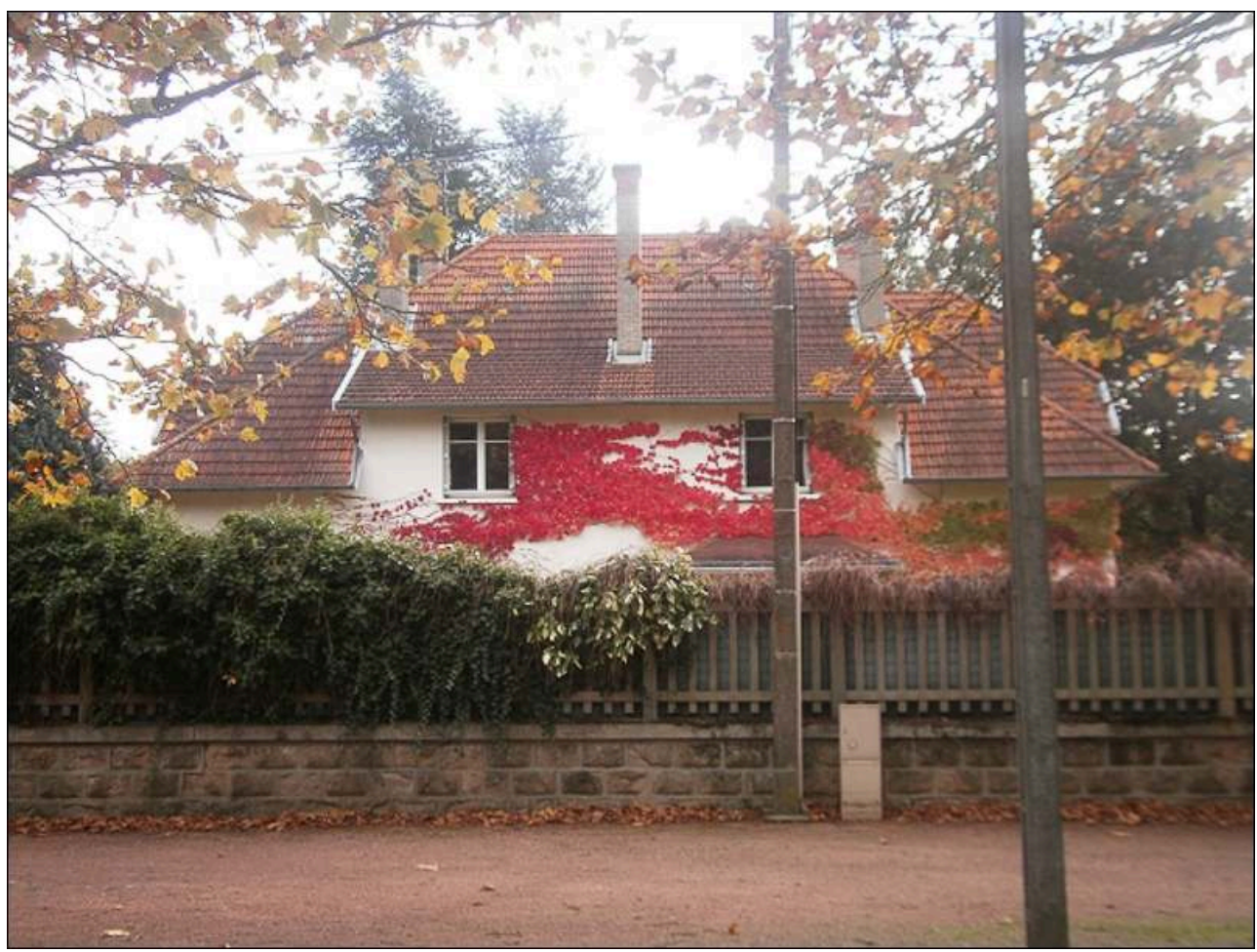

Auteur : D. Miralles Buil, octobre 2013.

Selon l'adjointe en charge de l'urbanisme, de l'habitat et du logement de la mairie de Roanne, le quartier de l'Arsenal est "un quartier où la municipalité intervient très peu ». Pourtant, comme ailleurs à Roanne, la municipalité organise un Conseil de Quartier pour les habitants du quartier de l'Arsenal, côté Roanne. Nous avons tenté d'obtenir un rendez-vous avec la vice-présidente de ce Conseil de Quartier qui s'avère être également l'Adjointe chargée de la jeunesse, de l'éducation populaire et de la vie associative de la ville de Roanne. Mais contre toute attente, celle-ci nous a répondu qu'elle ne pouvait absolument rien nous dire sur la question de la mémoire ouvrière et de l'éducation populaire du quartier, "car il n'y a rien à en dire ». Cette affirmation nous incite à penser que soit cette élue ignore l'histoire ouvrière du quartier de l'Arsenal, soit elle considère qu'elle n'est plus d'actualité. Quoi qu'il en soit, cette information nous renseigne sur l'image que se fait la mairie de Roanne du quartier de l'Arsenal et de la Cité Roanne.

Affirmation confirmée par la déclaration du responsable de la Direction Cadre de Vie du Service Urbanisme de Roanne, la Cité Roanne n'est pas une "cité ouvrière très commune ». De plus, il n'y a eu aucun règlement provenant de la mairie concernant les réhabilitations de ces logements. Toujours selon lui, de nombreuses extensions (cabanons, vérandas, etc.) ont vu le jour (comme en témoigne l'illustration 12 cidessous) et les habitants "ont fait ce qu'ils voulaient", et d'ajouter que "c'est un peu le bazar dans ce quartier ». Il s'agit pour lui d'un paysage urbain atypique qui ne correspond pas à l'architecture d'origine de la Cité. En conséquence, les « Maisons de l'Arsenal » ne sont absolument pas situées dans un périmètre de protection instauré par les Architectes des Bâtiments de France (ABF). Ainsi, il ne semble pas qu'il y ait réellement 
de prise en compte de ce patrimoine par la mairie de Roanne, ni architecturale, ni sociale, ni «mémorielle».

Illustration 12 - Une "Maison de l'Arsenal" de la Cité Roanne ayant bénéficié d'extensions et de réhabilitations

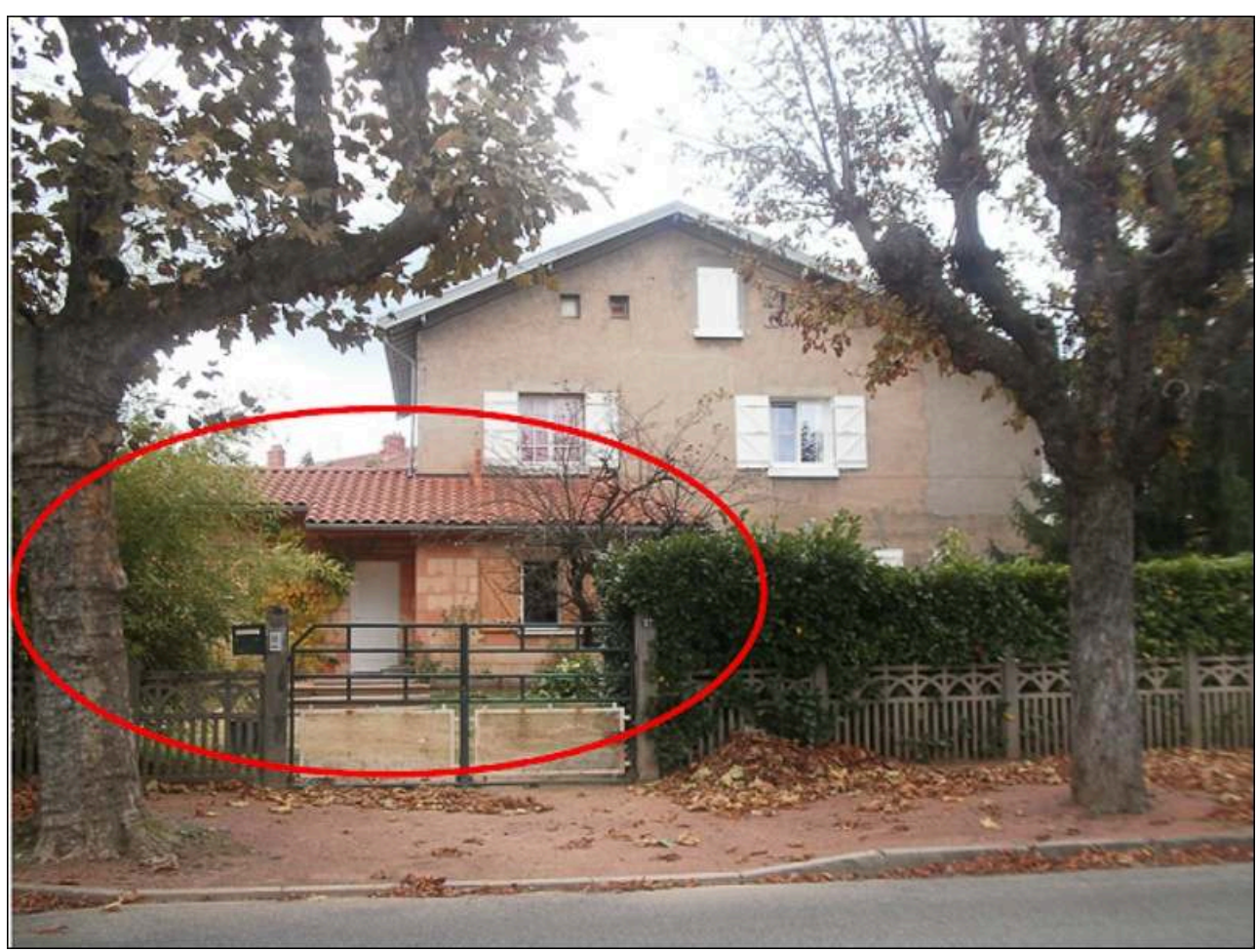

Auteur : D. Miralles Buil, octobre 2013.

On observe donc clairement une forte divergence entre la vision que portent les municipalités de Mably et de Roanne sur leurs cités ouvrières respectives. En effet, d'après le président de l'Amicale Laïque de l'Arsenal, la commune de Mably est aujourd'hui beaucoup plus active dans la valorisation de ce patrimoine industriel que la ville de Roanne, à la fois d'un point de vue matériel (l'architecture et l'aménagement du quartier) que immatériel (l'organisation sociale du quartier). Selon lui, «Roanne a des préoccupations plus urgentes que la valorisation ou le maintien de la mémoire ouvrière du quartier».

29 Mais comme pour la Cité Mably, on retrouve le même processus de brassage des populations d'origine de la Cité Roanne. De plus en plus d'anciens habitants quittent le quartier et sont remplacés par une population n'ayant pas vécu «le temps de l'Arsenal ». Néanmoins, ces nouvelles populations semblent plus aisées que celles de la Cité Mably, ce qui accroît encore le clivage social entre les deux entités anciennement réunies sous l'autorité de l'atelier de l'Arsenal. Effectivement, d'après un ancien technicien de l'Arsenal habitant la Cité Roanne depuis plus de trente-cinq ans, beaucoup d'habitants ont quitté les lieux et la majorité des "Maisons de l'Arsenal » sont aujourd'hui occupées par de nouveaux ménages appartement à la classe moyenne ou aisée de la population roannaise. "Les prix ont beaucoup augmenté depuis les années 2000. Nous avons rapidement eu de nouveaux voisins qui n'avaient rien à voir avec l'Arsenal et qui allaient travailler à Roanne ou ailleurs" indique-t-il. Ces nouveaux arrivant ne 
semblent pas prêts à renouer avec le passé ouvrier de ce «lieu à part » (Duchêne, 2005, p. 519).

Ces nouveaux venus ainsi que l'inactivité de la municipalité de Roanne concernant une possible valorisation de la Cité Roanne amènent à penser que cette dernière subit actuellement un fort processus de normalisation socio-spatiale tendant à en supprimer la spécificité historique.

\section{L'impossible patrimonialisation de la mémoire ouvrière des cités de l'Arsenal}

31 Lors de notre enquête de terrain, le terme de "patrimonialisation" n'a pas été prononcé par les habitants ou par les membres de la mairie de Mably ou de Roanne. Pourtant, comme nous l'avons vu précédemment, il est clair que le maintien de la mémoire ouvrière de la Cité Mably tient tout particulièrement à cœur à la municipalité de Mably. Ce n'est pas réellement l'aspect architectural que tente de valoriser la municipalité mais bien l'organisation sociale, cet entre soi. Tout comme le rapporte François Duchêne pour la Cité Berliet (Saint-Priest), c'est sur cette base que les " anciens habitants" de la cité ouvrière de l'Arsenal "tendent à ériger leur cité en patrimoine» (Duchêne, 2005). En effet, lors de notre terrain de recherche, la plupart des "anciens" de la cité ouvrière de Mably et de Roanne que nous avons rencontrés mettaient en évidence la spécificité de l'organisation sociale de cet "isolat urbain » (Duchêne, 2005). Pourtant, c'est avant tout la municipalité de Mably qui tente de favoriser ce maintien de la mémoire ouvrière véhiculée par la cité ouvrière de Mably, les habitants étant quant à eux davantage résignés à voir disparaître avec eux cette mémoire collective. La municipalité tente de susciter une mobilisation citoyenne autour de la cité ouvrière et de sa mémoire historique par le biais d'expositions et d'événements culturels. Mais malgré cela, on ne compte aujourd'hui aucune association de mémoire mise en place par les habitants du quartier. Le cas de la Cité Mably est donc bien un contre-exemple d'une mobilisation habitante réclamant la patrimonialisation d'un ensemble urbain. La disparition de la matérialité de cet objet-patrimonial - à savoir les logements ouvriers d'origine - peut expliquer cette indifférence face au projet de patrimonialisation de la part des habitants. En effet, ils ne semblent pas ressentir la nécessité de préserver une mémoire et une organisation ouvrière aujourd'hui révolue de par la rupture du lien social et économique avec l'Arsenal.

On peut supposer que la ville de Mably mobilise ici « l'argument patrimonial» dans une volonté de favoriser une plus grande cohésion socio-spatiale de la commune. En effet, la situation géographique et l'organisation spatiale de cette " petite ville " industrielle - comptant quatre foyers de population distincts et isolés les uns des autres - ont pu pousser la municipalité à valoriser chacun de ces quartiers afin qu'aucun d'entre eux ne se sente "extérieur" à Mably. Vincent Veschambre nous rappelle l'importance des enjeux sociaux et politiques qui peuvent se cacher «derrière la volonté de mettre en valeur et de conserver certains héritages, certains espaces» (Veschambre, 2007, p. 379). En effet, pour le cas de Mably on peut s'interroger sur la possible utilisation de l'argument patrimonial par la municipalité dans le but de renforcer son ancrage et son influence sur ce quartier limitrophe de la ville de Roanne.

De l'autre côté de la limite communale, la municipalité de Roanne ne semble pas sur la même longueur d'onde que son homologue mablyrote. Nous l'avons vu, pour elle « il n'y 
a rien à dire " concernant la mémoire ouvrière de la Cité de l'Arsenal, qui ne semble donc pas valorisée. Inversement, elle essaie d'intégrer ce quartier en le rendant par exemple très accessible depuis le centre-ville de Roanne (voir l'illustration 3 et la ligne de bus reliant directement le quartier de l'Arsenal au centre-ville de Roanne). On sait que la forte distinction d'un quartier peut gêner une commune dans ses projets urbains car elle peut engendrer de forts mouvements sociaux de populations revendiquant par exemple des aides à la réhabilitation de leurs logements. En effet, la valorisation ou la patrimonialisation d'ensembles urbains sont des concepts "éminemment conflictuels" (Garnier, Castrillo Romón, 2013). Autour de ceux-ci peuvent se cristalliser des processus sociaux de revendication et d'appropriation de l'espace (Ripoll, Veschambre, 2006). C'est selon nous la raison pour laquelle la ville de Roanne essaie de mettre en place des processus actifs de normalisation de ses quartiers ouvriers.

Nous assistons donc à un processus de valorisation/normalisation paradoxal. Du côté de Mably, les cités ouvrières ne sont pas d'origine, mais la municipalité tente tout de même de valoriser ce patrimoine industriel, historique et social spécifique; et de l'autre, la municipalité de Roanne ne semble pas réellement valoriser ses « Maisons de l'Arsenal » qui datent pourtant de 1917 et témoignent encore d'une architecture et d'une histoire ouvrière sombrant chaque jour davantage dans l'oubli.

\section{Conclusion : la patrimonialisation face aux enjeux territoriaux des communes}

Ainsi, les « enjeux patrimoniaux » ne sont pas les mêmes pour les deux communes et entraînent donc un traitement différencié des cités ouvrières de l'Arsenal. Nous avons vu que, concernant la possible patrimonialisation de la Cité Mably, la "participation habitante » fortement espérée par la municipalité ne fait pas du tout consensus parmi les habitants de Mably. Inversement, la ville de Roanne semble vouloir se démarquer de son image passéiste de ville industrielle. Elle mise aujourd'hui davantage sur une politique d'attractivité basée sur la gastronomie et la culture et ne tente pas de valoriser son patrimoine ouvrier. Les territoires de Mably et de Roanne ne disposant pas des mêmes enjeux économique et sociaux, les stratégies de valorisation/ normalisation de leurs cités ouvrières, bien que connexes, divergent donc fortement.

On comprend bien qu'en matière de patrimonialisation, les modalités d'actions et de réactions des municipalités (et plus largement des institutions patrimoniales) face à l'action ou l'inaction des habitants varient fortement selon les contextes locaux et les enjeux de ces territoires. De plus, à l'heure de la métropolisation et de la promotion généralisée de la mixité sociale par les politiques urbaines, il reste dans ces cités ouvrières de moins en moins d'habitants susceptibles de prendre en main une quelconque «mobilisation patrimoniale ». Si l'on ajoute à cela le contexte économique de ces espaces urbains périphériques et la vétusté de leurs bâtiments, on comprend bien que ces cités faiblement attractives ne possèdent pas les ressources nécessaires à leur patrimonialisation.

37 Cette étude de cas permet donc de s'interroger sur la place de la « fabrique patrimoniale » au sein des villes moyennes françaises. En effet, contrairement aux grandes villes industrielles, les villes moyennes ne disposent généralement pas des moyens institutionnels et financiers nécessaires à la patrimonialisation de leurs 
"héritages matériels ou immatériels" (Veschambre, 2007, p. 362). On peut donc s'interroger sur l'avenir des cités ouvrières dans les villes moyennes et les "petites villes » industrielles françaises.

\section{BIBLIOGRAPHY}

Barras M., 1998. Histoire de l'Arsenal de Roanne. Roanne, Éditions lyonnaises d'art et d'histoire, $350 \mathrm{p}$.

Coanus T., 1995. Paysage, risques naturels et intérêts locaux : Un conflit de représentations en moyenne montagne (Sainte-Foy-Tarentaise, Savoie). In Claudie V. (dir.), Paysage au pluriel : Pour une approche ethnologique des paysages. Paris, Éditions de la Maison des sciences de l'homme (Ethnologie de la France), p. 134-149.

Debos F., 2012. Le développement du tourisme : facteur de valorisation du patrimoine industriel ?, Séminaire international, Processus, problématiques, enjeux du patrimoine industriel, du 21 au 24 juin 2012 à Baia-Maré (Roumanie).URL : http://archivesic.ccsd.cnrs.fr/ sic_00743818. Consulté le 3 décembre 2014.

Duchêne F., 2005. Les Anciennes Cités ouvrières, entre patrimonialisation et normalisation. In Gravari-Barbas M. (dir.), Habiter le patrimoine. Enjeux, approches, vécu. Rennes, Presses universitaires de Rennes, p. 517-526.

Duchêne F., 2010a. Perspectives historiques d'un modèle de logements patronaux : entre hygiénisme et références coloniales, les cités ouvrières. In Duchêne F. (dir.), Cités ouvrières en devenir. Ethnographies d'anciennes enclaves industrielles. Saint-Etienne, Publications de l'Université de Saint-Etienne, p. 19-41.

Duchêne, F., 2010b. La double normalisation, sociale et urbaine, du quartier des cités à Roussillon et Salaise-sur-Sanne, dans l'Isère. In Duchêne F. (dir.), Cités ouvrières en devenir. Ethnographies d'anciennes enclaves industrielles. Saint-Etienne, Publications de l'Université de Saint-Etienne, p. 151-181.

Duchêne F., Langumier J., Morel Journel C., 2010. Introduction. In Duchêne F. (dir.), Cités ouvrières en devenir. Ethnographies d'anciennes enclaves industrielles. Saint-Etienne, Publications de l'Université de Saint-Etienne, p. 9-16.

Duchêne F., Langumier J., Morel Journel C., 2013. Cités ouvrières et patrimonialisation : d'un modèle à ses multiples transformations. Espaces et sociétés, vol. 1, n 152-153, p. 35-50.

Garnier, J.-P., Castrillo Romón, M., 2013. Éditorial. Espaces et sociétés, Vol. 1, n¹52-153, p. 7-17.

Halbwachs M., 1997. La mémoire collective. Nouvelle édition revue et augmentée, Paris, Albin Michel, $295 \mathrm{p}$.

Houssel J.-P., 1979. Le Roannais et le Haut-Beaujolais textile, un espace à l'écart des métropoles. Lyon, Presses Universitaires de Lyon, $230 \mathrm{p}$. 
Le Pays Roannais, 29 août 2013. Retour sur l'histoire du quartier de l'Arsenal. URL : http:// www.lepaysroannais.fr/region/edition/roanne/roanne-roanne/2013/08/29/retour-sur-lhistoiredu-quartier-de-larsenal_1671892.html. Consulté le 10 avril 2015.

Morel Journel C., 2010. De la maison ouvrière au pavillon : quand les « signes des temps miniers » s'estompent à la cité des Combes. In Duchêne F. (dir.), Cités ouvrières en devenir. Ethnographies d'anciennes enclaves industrielles. Saint-Etienne, Publications de l'Université de Saint-Etienne, p. 127-149.

Ripoll F., Veschambre V., 2006. L'appropriation de l'espace : une problématique centrale pour la géographie sociale. In Séchet R. (dir.), Penser et faire la géographie sociale : Contribution à une épistémologie de la géographie sociale. Rennes, Presses universitaires de Rennes, p. 295-304.

Rocher J.-L., Trévarin G., 2011. Mably, terre d'accueil, d'art et de solidarité. Roanne, Thoba's éditions, $208 \mathrm{p}$.

Vanier M., 2006. Partager la souveraineté territoriale : objets, modes et principes de l'État «glocal ». Rives méditerranéennes, $n^{\circ} 25$, p. 9-18.

Veschambre V., 2002. Une mémoire urbaine socialement sélective. Réflexions à partir de l'exemple d'Angers. Annales de la Recherche Urbaine, nº 92, p. 65-73.

Veschambre V., 2005. Effacement et réappropriation de l'habitat populaire dans les centres anciens patrimonialisés : les exemples du Vieux-Mans et de la Doutre à Angers. In Gravari-Barbas M. (dir.), Habiter le patrimoine : Enjeux, approches, vécu. Rennes, Presses universitaires de Rennes, p. 245-264.

Veschambre V., 2007. Patrimoine : un objet révélateur des évolutions de la géographie et de sa place dans les sciences sociales. Annales de géographie, vol. 4, n656, p. 361-381.

Zanetti T., 2012. Une ville et sa multinationale, une multinationale et sa ville : emprise spatiale, organisation sociale, fonction économique et régulation politique du «territoire Michelin » à Clermont-Ferrand (fin XIXème à nos jours). Thèse de doctorat en géographie, aménagement et urbanisme, Université Lumière Lyon 2, 837 p.

Sitographie

Site internet de la Ville de Mably. Consulté le 10 avril 2015. http://www.ville-mably.fr/ presentation.html

Site internet de la Ville de Roanne. Consulté le 10 avril 2015. http://www.roanne.fr/37-letextile.htm

\section{NOTES}

1. L'aspect inachevé de cette tentation patrimoniale provient également du fait que ces cités sont toujours habitées par des familles populaires, elles n'ont donc pas réellement changé de fonction (Duchêne, 2005, p. 525).

2. Cet article s'appuie sur un stage de terrain réalisé en Master 1 Géographie, Environnement, Territoire en 2013 à l'Université Lumière Lyon 2. Au cours de cette étude, des entretiens semidirectifs ont été réalisés avec le maire et le Conseiller délégué à l'habitat de la commune de Mably ; le responsable de la Direction Cadre de Vie du Service Urbanisme et l'Adjointe en charge de l'urbanisme, de l'habitat, du logement, des relations européennes et internationales de la ville de Roanne; le co-auteur du livre Mably terre d'accueil, d'art et de solidarité (2011) ainsi que le président de l'Amicale Laïque de l'Arsenal (ALA). Concernant les habitants des cités ouvrières de 
l'Arsenal, des entretiens non-directifs ont été réalisés avec sept habitants de l'ancienne Cité Mably, quatre de l'ancienne Cité Roanne et trois habitants des anciens camps situés entre les deux anciennes Cités ouvrières. La durée des entretiens semi-directifs variant de 20 minutes à 1 heure 30 et celle des entretiens non-directifs de 10 à 30 minutes environ.

3. À l'instar du «territoire Michelin » élaboré par Thomas Zanetti lors de sa thèse, il est possible d'appréhender un «territoire de l'Arsenal ", vu comme "un construit spatial, social, économique est politique formant un millefeuille» et représentant "une entité socio-spatiale formée par les unités résidentielles et les équipements collectifs construits par l'entreprise et destinés à ses salariés » (Zanetti, 2012, p. 15).

4. En plus des cités ouvrières de Mably (au nord) et de Roanne (au sud), deux camps - dits du Colombier et des Essarts - logeant un millier de célibataires et une centaine de ménages, ajoutaient encore une touche de diversité au tableau des cités ouvrières de l'Arsenal. Ces trois entités disparates se retrouvent clairement sur les plans reproduits lors d'une exposition réalisée en 2008 par la médiathèque de Mably. Voir le plan de l'Arsenal et de ses cités : http://www.villemably.fr/tl_files/pdf/ville/plan-arsenal.pdf, consulté le 10 avril 2015.

5. Néanmoins, à cette époque, bien que les travailleurs de l'Arsenal habitant dans ces nouveaux lotissements fussent propriétaires de leur habitation, le lien entre l'Arsenal et les logements n'était pas brisé. En effet, seuls les travailleurs de l'Arsenal pouvaient obtenir un logement au sein du « territoire de l'Arsenal ».

6. François Duchêne témoigne bien de cette «disparition" des cités ouvrières du fait de l'émergence des HLM dans le cas des « anciennes cités cheminotes de la région lyonnaise » (Duchêne, Langumier, Morel Journel, 2013, p. 43-44).

7. Voir l'exposition réalisée en 2008 sur le site internet de la ville de Mably: http://www.villemably.fr/tl_files/pdf/ville/exposition\%20arsenal\%20et\%20cites\%20a\%20mably.pdf, consulté le 10 avril 2015.

8. On peut bien sûr le trouver à la bibliothèque municipale de Mably, de Roanne, mais aussi à Lyon ou à Paris.

9. Pour élaborer cette illustration, nous avons réalisé une mosaïque avec les anciennes photographies aérienne de l'IGN ainsi qu'avec l'aide d'un logiciel SIG afin de géoréférencer ces «dalles photographiques » et d'y superposer le réseau viaire actuel préalablement digitalisé sur un SIG.

\section{ABSTRACTS}

The working-class housing of Arsenal is located in the Loire department (France) between two towns: Roanne and Mably. However, the two municipalities don't have the same stance regarding the social and spatial processes that the city is subjected to nowadays. Using this case study, this article's goal is to show how municipalities can seize the concept of patrimonialization in order to make the area more attractive using a "territorial marketing" approach. It studies the attempts to create a "patrimonial drive" that a municipality may encourage, the limits of the notion of "resident participation" in the development of a patrimonialization process of theses "urban isolates". Finally, this document reflects on the future of theses urban forms in French medium-sized towns. 
La cité ouvrière de l'Arsenal, dans le département de la Loire, est située entre les deux communes de Roanne et de Mably. Or, les deux municipalités n'ont pas la même attitude face aux processus socio-spatiaux que subit aujourd'hui cette cité ouvrière. À partir de ce cas d'étude, cet article souhaite montrer comment les municipalités peuvent s'emparer du concept de patrimonialisation afin de valoriser leur territoire, dans une logique de « marketing territorial». Il aborde les tentatives de création d'une «volonté patrimoniale » qu'une municipalité peut tenter d'impulser sur sa commune et analyse les limites de la notion de "participation habitante " dans l'élaboration d'un processus de patrimonialisation de ces "isolats urbains". Enfin, il questionne le devenir de ces formes urbaines situées dans les villes moyennes françaises.

\section{INDEX}

Mots-clés: patrimonialisation, cité ouvrière, Rhône-Alpes, normalisation, mémoire ouvrière Keywords: patrimonialization, working-class housing, Rhône-Alpes, normalization, workingclass history

Subjects: Sur le Champ - Sur le Terrain

\section{AUTHOR}

\section{DIEGO MIRALLES BUIL}

Diego Miralles Buil, diego.miralles@univ-lyon2.fr, est doctorant en Géographie à l'Université Lumière Lyon 2, UMR 5600 CNRS Environnement Ville Société. Il a publié récemment :

- Miralles Buil D., 2015. Les coopératives d'habitation en cession d'usage à Barcelone. La réémergence de l'habitat alternatif comme solution viable face à la crise du logement à Barcelone ? Mémoire de Master 2 de géographie, Université Lumière Lyon 2, $221 \mathrm{p}$.

- Miralles Buil D., 2014. Calafou, une coopérative d'habitants en devenir. Mémoire de Master 1 de géographie, Université Lumière Lyon 2, 210 p. 